\title{
Sentinel Lymph Node Biopsy in Early Breast Cancer Using the Indocyanine Green Fluorescence Technique: A Validation Study
}

\author{
Loh SK ${ }^{a}$, Baharudin $S^{a}$, A. Latiff J ${ }^{a}$, Mahamad Dom SA ${ }^{b}$, Suhaimi $S^{c}{ }^{c}$ \\ ${ }^{a}$ Department of Surgery, Hospital Selayang, B21 - Lebuhraya Selayang - Kepong, 68100 Batu Caves, Selangor, Malaysia \\ ${ }^{b}$ Department of Pathology, Hospital Selayang, B21 - Lebuhraya Selayang - Kepong, 68100 Batu Caves, Selangor, Malaysia \\ ${ }^{c}$ Department of Surgery, UKM Medical Center, Jalan Yaakob Latiff, 56000 Cheras, Kuala Lumpur, Malaysia
}

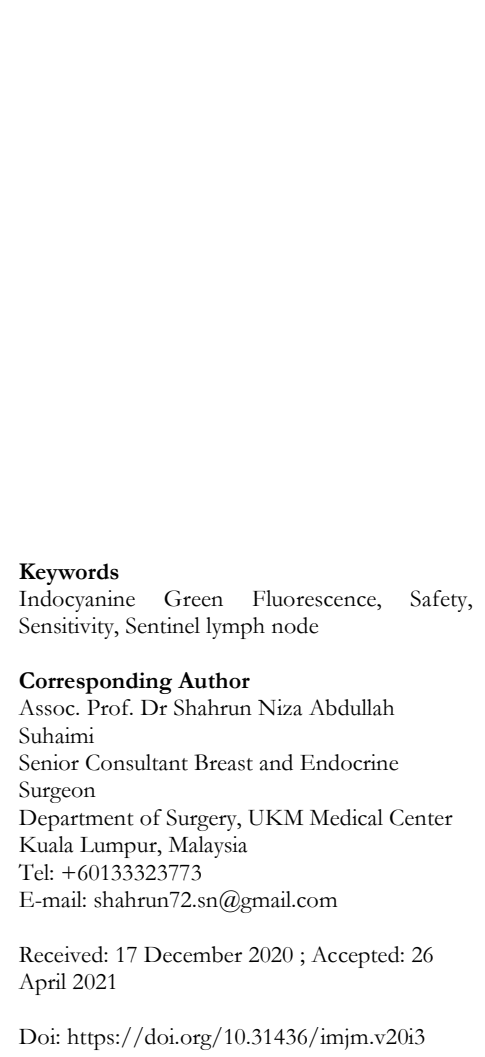

\section{ABSTRACT}

INTRODUCTION: Introduction: Sentinel lymph node biopsy (SLNB) is now recognized as the standard of care for early breast cancer patients with negative axillary lymph nodes. Various approaches for Sentinel Lymph Node (SLN) identification using either the blue dye method or scintigraphy alone or their combination have been proposed. However, this method is costly and may not be applicable in certain developing countries. SLNB involving the use of indocyanine green (ICG) offers several advantages, and it is valid and safe when in direct comparison with the blue dye method and scintigraphy. Hence, we performed SLNB using this method in early breast cancer as the first center that involves the use of ICG in Malaysia. We performed validation study on this method with the aims to determine its sensitivity and safety profile. MATERIALS AND METHODS: This is a validation and non-randomised prospective observational study involving 20 patients underwent SLNB wherein ICG is used for localisation. The patients were recruited according to the recommendations stipulated in the Malaysia Clinical Practice Guideline. RESULT: The average number of SLNs removed per patient was 4.0 (range, 3-6) with sentinel lymph nodes detection rate at $98.75 \%(79 / 80)$. The false negative rate is at $5 \%$. No adverse events were observed in all cases. CONCLUSION: The ICG fluorescence method is simple, reliable and safe. Moreover, it demonstrates a high SLN detection rate with a low false-negative rate, and it does not require a special instrument for radioisotope use.

\section{INTRODUCTION}

Approximately 1.7 million cases annually were diagnosed with breast cancer hence makingit the most common cancer in females globally.1 Axillary lymph node management in early breast cancer is important clinically as it depends on the lymph nodes status that receive metastases from the breast. Hence the early axillary management provides information on staging and prognosis as well as the implications for the locoregional control of breastcancer. ${ }^{2-3}$

Traditionally, axillary lymph node dissection (ALND) is the standard approach for axillary staging and in acquiring complete qualitative and quantitative information about breast cancer patients. However, this approach is linked with significant morbidities, such as seroma, lymphedema, shoulder stiffness and damage to motor and sensory nerves. ${ }^{4-6}$ To reduce the complications of ALND, sentinel lymph node biopsy (SLNB) can be used as an alternative option for axillary lymph node staging in early breast cancer.

In the National Surgical Adjuvant Breast and Bowel Project (NSABP) B-32 trial, more than 5,000 sentinel lymph node (SLN) biopsy-negative patients were randomised into the no further surgery group and the ALND group. Results showed no significant differences between these groups in terms of overall survival, disease-free survival or local-regional recurrence7. 
Therefore, SLNB is currently recognized as the standard of care for patients with negative axillary lymph nodes shown during clinical and radiological evaluation.

Various approaches for SLN detection using either the blue dye method or scintigraphy alone or their combination have been proposed.8-9 According to the ASCO guideline, the combination of radioisotope and blue dye results in high identification rate and low false - negative rate10. However, despite its effectiveness in SLN detection, scintigraphy potentially exposes patients and healthcare workers to radioisotopes. Moreover, this method is costly and it requires an operating license, specialized instruments, and centres with experiences with the use of the said technique. As a result, this method is not widely practiced in Malaysia.

The development of new efficient techniques for SLNB has become popular. Studies have shown that SLNB involving the use of indocyanine green (ICG) offers several advantages, and it is valid and safe when in direct comparison with the blue dye method and radioisotope method.11-16 Also, these studies have shown that when used in SLN identification, ICG demonstrated a high and reliable sensitivity ranging from $89 \%$ to $100 \% .11-16$

ICG is a popular reagent that has been approved for clinical usage. It absorbs light in the near infra-red range $(800 \mathrm{~nm})$, and it emits fluorescence when it comes into contact with plasma proteins. The fluorescence signal is captured by a photodynamic eye camera and then converted into a black and white image that is displayed on a TV monitor.16

Studies have shown that ICG is safe when administered through intradermal or intravenous injection and causes no major side effects.14,16,17 In these studies, only mild to moderate adverse events, such as tachycardia, vomiting and nausea, were recorded, and no therapeutic consequences for such reactions were observed during the ICG toxicity assessment.14,16,17 Besides that, no local skin allergic reaction or permanent skin staining were observed. In view of the high sensitivity of ICG in SLN identification and given the proven safe use of ICG in SLNB, the objective of our study is to determine the sensitivity and safety of SLNB in our center as the first that involves the use of ICG in Malaysia.

\section{MATERIALS AND METHODS}

\section{Study Design}

The study was a validation, non-randomized, prospective, observational study involving 20 patients scheduled to undergo SLNB wherein ICG was used for localization. The patients selected for SLNB were recruited according to the recommendations stipulated in the Malaysia Clinical Practice Guideline.

All patients were only recruited once they had signed their informed consent formed prior to participation in the study. The study was performed according to the ethical standard of the Declaration of Helsinski and had received an approval from the local ethical research committee of National Medical Research Register (NMRR-18-3210-44275) prior to conduct of the study.

The sensitivity of ICG is based on the amount of detected fluorescent-stained SLNs. This meant that only the nodes that were fluorescent were considered as sentinel nodes. Sentinel lymph node with histologically absent tumor deposits while other axillary nodes containing tumor deposits are considered to be falsenegative sentinel node. This study is not industrial sponsored.

\section{Inclusion and Exclusion Criteria}

The patients recruited in this study were aged 18 years and above, with early breast cancer according to The American Joint Committee on Cancer (AJCC) Cancer Staging Manual with clinically negative nodes (both clinical examination and axillary imaging). Patients with a history of axillary surgery, had received neo-adjuvant chemotherapy/radiotherapy, pregnantor lactating, with a history of hypersensitivity to iodine/ICG, and underwent immediate breast reconstruction surgery were excluded. 


\section{Histopathology of SLNs}

All SLNs underwent pathological evaluation according to our hospital protocol. Haematoxylin and eosin (H\&E) staining is the most common technique used to demonstrate clearly the morphological tissue structures. Haematoxylin stains cell nuclei blue whereas eosin stains the cytoplasm and most connective tissue fibres with varying shades and intensities of pink, orange and red. The equipment used were as follows: Shandon Varistain, Memmert hot air oven and slide racks. All solutions are commercially prepared (haematoxylin, eosin, clarifier and bluing reagent), and 70\% alcohol

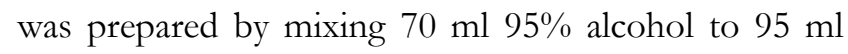
distilled water.

- Macrometastasis is defined as a metastasis in the lymph node(s) measuring at least $1 \mathrm{~mm}$ and its greatest dimension is larger than $2 \mathrm{~mm}$.

- Micrometastasis is defined as a metastasis in the lymph node(s) measuring not larger than $0.2 \mathrm{~mm}$ and/or comprising more than 200 cells.

- Isolated tumour cells (ITC) are single tumour cells or small clusters of cells not more than $0.2 \mathrm{~mm}$ in its greatest dimension and can be detected by routine $\mathrm{H} \& \mathrm{E}$ orimmunohistochemistry.

- Additional criterion includes a cluster of fewer than 200 cells in a single histological crosssection.

\section{Operative Techniques}

After induction, $2 \mathrm{ml}$ 0.5\% ICG (Aurogreen, Lyophilized $25 \mathrm{mg}$ Indocyanine Green, Aurolab, India) was administered through subcutaneous and intradermal injection using a 23 gauge needle into the periareolar region after the sterilization of the operative site. After the injection of ICG into the edge of the areola, the breast was massaged for 2 min. A skin incision was made over the lower axillary line and then the axillary space was probed.

The ICG-stained SLNs were identified with a KARL STORZ ICG system (Image1 spiesTM camera system, HOPKINS ${ }^{\circledR}$ Forward-Oblique Telescope 30o, and Cold Light Fountain D-LIGHT P SCB) and removed under the real-time imaging guidance of a monitor. The
SLNs were considered clear once no ICG-stained node could be further identified in the axillary space by theoperating surgeon. All the excised nodes were classified and recorded as fluorescence positive or negative.

Operations were performed according to our preoperative planning and surgical approaches, including mastectomy, breast-conservation surgery or nipplesparing mastectomy, followed by a standard axillary clearance. ICG does not contain any known metabolites, and it is extracted by the liver and then incorporated into the bile juice. 17 Therefore, the immediate adverse reaction of ICG was recorded intraoperatively, and the patients were followed up until they were discharged from the hospital.

\section{Flow Chart of The Operative Techniques}

$2 \mathrm{ml} \mathrm{0.5 \%} \mathrm{ICG} \mathrm{was} \mathrm{administered} \mathrm{through} \mathrm{subcutaneous} \mathrm{or} \mathrm{intradermal}$ injection at the periareolar region using 23-gauge needle; a 2-minute massage was given following the induction. Skin incision was made at the lower axillary line. Sentinel lymph node was identified and removed with KARL STORZ system under real-time imaging guidance. The SLNB was completed when no more ICG-stained node could be identified by the operating surgeon.

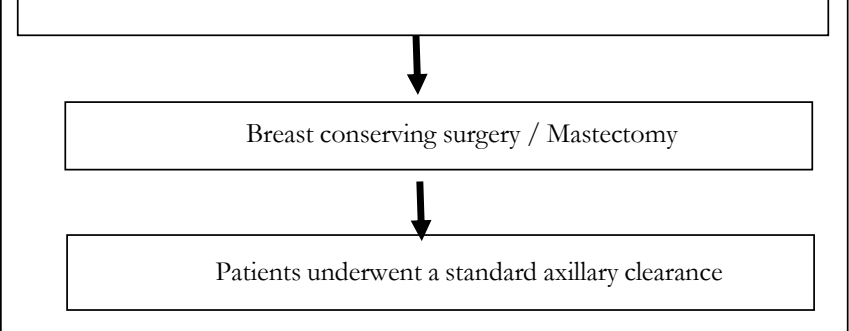

Figure 1 Flow chart of the operative technique

\section{RESULTS}

\section{Patient and Tumor Characteristics}

Twenty women with mean age of 54 years (range, 40-79 years) with an average tumor size of $25.2 \pm 9.5 \mathrm{~mm}$ were included in this study. Overall, 95\% of them (19 of 20)had an invasive ductal carcinoma and 5\% (1 of 20) had a high-grade ductal carcinoma in-situ (DCIS), and they were all recommended to undergo SLN biopsy. The clinical pathological characteristics of the patients are summarised in Table I. 
Table I Tumour characteristics

\begin{tabular}{|c|c|c|c|}
\hline & & $\begin{array}{l}\text { Number of } \\
\text { patients }\end{array}$ & $\%$ \\
\hline \multirow[t]{2}{*}{ Histology } & DCIS & 1 & 5.0 \\
\hline & IDC & 19 & 95.0 \\
\hline \multirow[t]{3}{*}{ Grade } & I & 2 & 10.0 \\
\hline & II & 12 & 60.0 \\
\hline & III & 5 & 25.0 \\
\hline \multirow[t]{2}{*}{ ER } & Negative & 3 & 15.0 \\
\hline & Positive & 17 & 85.0 \\
\hline \multirow[t]{2}{*}{ PR } & Negative & 5 & 25.0 \\
\hline & Positive & 15 & 75.0 \\
\hline \multirow[t]{2}{*}{ HER2 } & Negative & 16 & 80.0 \\
\hline & Positive & 4 & 20.0 \\
\hline
\end{tabular}

DCIS $=$ ductal carcinoma in-situ, IDC=invasive ductal carcinoma Grade $=$ Scarff-Bloom-Richardson grading system

$\mathrm{ER}=$ estrogen receptor $\mathrm{PR}=$ progesterone receptor

HER2=human epidermal growth factor receptor 2 Sentinel lymph node biopsy

Eighty nodes were retrieved and evaluated using the ICG technique. The average number of SLNs removed per patient was 4.0 (range, 3-6) (Table II). The node detection rate when using ICG was $98.75 \%$ (79/80). Positive SLN was found in 7 patients (35\%), and metastases were found in 11 nodes, all of which were fluorescent-stained. In one patient, the SLN detected with ICG showed no metastasis; however, the final histology from axillary clearance nodes had presence of metastasis. Hence, the false-negative rate of ICG was $5 \%$. Moreover, no adverse reactions were recorded in any of the 20 patients during surgery. No skin tattooing or allergic reactions/ necrosis of the skin were observed after the intradermal injection of ICG.

Table II SLNB result

\begin{tabular}{lcc}
\hline & $\begin{array}{c}\text { Number of patients/ } \\
\text { Nodes }\end{array}$ & $\%$ \\
\hline Average number of SLN nodes removed & 4.0 & - \\
Detection rate of ICG & $79 / 80$ & 5 \\
False-negative rate for SLN when using ICG & $1 / 20$ & 5 \\
Total number of SLNs containing metastasis & 11 & 0 \\
Adverse reaction of ICG & 0 & \\
\hline
\end{tabular}

\section{DISCUSSION}

SLNB is a widely accepted substitute to ALND. Standard axillary clearance may beavoided in patients without SLN involvement in order to decrease the morbidity factors related to ALND., 4, 18 Technetium-99 (99mTc) sulphur colloids and $99 \mathrm{mTc}$ albumin colloids are frequently used globally for SLN detection. Gamma probe is used to detect these radiocolloids once they are trapped in the SLNs. The combination of radioisotope and blue dye can decrease false negative rates.19 However, special training is necessary for this method in a facility specialized in dealing with radioisotopes.

The ICG fluorescence techniqueis a modification of the dye method, and it allows a surgeon to explore the axilla through the fluoroscent lymphatic vessels to detect the SLNs. In our study, data from 20 early breast cancer patients were analysed. The SLN detection rate was $98.75 \%(79 / 80)$ which is similar to the rate achieved with the method involving the combined use of radioisotope and dye.20-23 Besides the accuracy of the SLN detection using ICG, the false-negative rate is also important in determining the clinical efficacy of this technique. In our study, we reported a false- negative rate of $5 \%$ which is comparable with the false-negative rates ranging from $2 \%$ to $22 \%$ in large series. ${ }^{24-26}$

The radioisotope method allows exploration to the skin incision site through radiation measurement, meanwhile the ICG fluorescence technique can specifically identify a skin incision site by detecting the lymphatic vessels of the skin. The radioisotope method is quantitative as first node removal is confirmed when less than $10 \%$ of the nodes remain in the operation site by using a radioisotope tracer.27 Furthermore, this method allows pre- operative of SLN quantification prior to surgery through lymphoscintigraphy.

However, fluorescence technique provides real time imaging over the axillary space. This meant that the amount of lymph nodes to be removed depends on the expertise of the surgeon to detect the fluorescentstained nodes under direct vision. Besides that, no evidence shows whether the 'hottest' node in the radioisotope method is identical to a maximally fluorescentnode in the ICG method. In our study, the mean number of SLNs removed per patient was 4.0 which is larger than that reported for the radioisotope technique.

In the study of Hojo et al., the average numbers of 
SLNs were 3.8 (fluorescence), 1.9 (blue dye only) and 2.0 (radioisotope only).28 The average number of removed lymph nodes is higher in the ICG fluorescence technique because it can detect paralymphatic tissue as well as SLNs. Based on our experience, higher node yield is also because of ICG leakage upon nodal dissection that led to non-specific staining towards the adjacent non-sentinel lymph nodes. In general, the ICG technique could attain a high detection rate similar to the radioisotope technique, but it cannot replace radioisotope technique until these two techniques can be compared directly.

The strongest fluorescent signals were detected within the $0.5-1 \mu \mathrm{g} / \mathrm{ml}$ concentration preclinically. At values different from this concentration range, the strength of the fluorescence signals will reduce. When the amount of body fluids is taken into consideration, injecting $5 \mathrm{mg}$ of ICG is favourable based on osmotic and hydrostatic pressures. Study showed the viewing of lymphatic flow depends on the dose given.29 Murawa et al reported that administration of $15 \mathrm{mg} / \mathrm{ml}$ ICG provides the best visualisation; however, SLNs detectionwas comparable when both 5 and $10 \mathrm{mg}$ of ICG was given. Another study reported that giving $0.25 \mathrm{mg} / \mathrm{ml}$ of ICG is adequate to identify SLNs and subcutaneous lymphatic flow.11 In our study, we used $10 \mathrm{mg}$ of ICG, which is $1 \mathrm{ml} / 5 \mathrm{mg}$ in concentration, as the optimal dose for the identification of SLNs and subcutaneous lymphatic flow. The optimal dose for ICG remains uncertain, and it relies on the administration volume and concentration of ICG. Therefore, further clinical trials that could evaluate the efficacy of ICG are needed.

\section{CONCLUSION}

The ICG fluorescence method is simple, reliable and safe as proven from the results of our study. It shows $98.75 \%$ SLN detection rate with $5 \%$ of false negative rate and no adverse reactions detected.

\section{ACKNOWLEDGEMENT}

We would like to thank Dr Suraya and Dr Juliana as the operating breast consultant in our study. Also much appreciation to Hospital Selayang for being the study site and Universiti Hospital Kebangsaan Malaysia for guidance.

\section{REFERENCES}

1. Torres LA, Bray F, Siegel RL, Ferlay J, LortetTieulent K, et al. Global cancer statistics, 2012. CA Cancer J Clin 2015; 65: 87-108.

2. Wang YL, Dong HY, Wu HY, Zhang L, Yuan K, Chen HQ, et al. Improved false negative rateof axillary status using sentinel lymph node biopsy and ultrasound suspicious lymph nodesampling in patients with early breast cancer. BMC Cancer 2015; 15: 382.

3. Ciatto S, Brancato B, Risso G, Ambrogetti D, Bukgaresi P, Maddau C, et al. Accuracy of fine needle aspiration cytology (FNAC) of axillary lymph nodes as triage test in breast cancer staging. Breast Cancer Res Treat 2007; 103: 8591.

4. Giuliano AE, Hunt KK, Ballman KV, Beitsch PD, Whitwirth PW, Blumencranz PW, et al. Axillary dissection vs no axillary dissection in women with invasive breast cancer and sentinel lymph node metastasis: a randomized clinical trial. JAMA 2011; 305: 569-75.

5. Lyman GH, Temin S, Edge SB, Newman LA, Turner RR, Weaver DL, et al. Sentinel lymph node biopsy for patient with early-stage breast cancer: American Society of ClinicalOncology clinical practice guideline update. J Clin Oncol 2014; 32: 1365-83.

6. Galimberti V, Cole BF, Zurrida S, Viale G, Luini A, Veronesi P, et al. Axillary dissection versus no axillary dissection in patients with sentinel-node micrometastases (IBCBG 23-01): a phase 3 randomised controlled trial. Lancet Oncol 2013; 14(4): 297-305.

7. Krag DN, Anderson SJ, Julian TB, Brown AM, Harlow SP, Costantino JP, et al. Sentinel- lymphnode resection compared with conventional axillary-lymph-node dissection inclinically nodenegative patients with breast cancer: overall survival findings from the NSABP B-32 randomised phase 3 trial. Lancer Oncol 2010; 11 (10): 927-33.

8. Thevarajah S, Huston TL, Simmons RM. A comparison of the adverse reactions associated 
with isosulfan blue versus methylene blue dye in sentinel lymph node biopsy for breast cancer.

Am J Surg 2005; 189(2): 236-9.

9. Ahmed M, Purushotham AD, Douek M. Novel techniques for sentinel lymph node biopsy in breast cancer: a systematic review. Lancet Oncol 2014; 15(8): e351-62.

10. Lyman GH, Guiliano AE, Somerfield MR, Benson AB 3rd, Borduka DC, Burstein HJ, et al. American Society of Clinical Oncology guideline recommendations for sentinel lymph node biopsy in early-stage breast cancer. J Clin Oncol 2005; 23: 7703-7720.

11. Sugie T, Kassim KA, Takeuchi M, Hashimoto T, Yamagami K, Masai Y, et al. A novel methodfor sentinel lymph node biopsy by indocyanine green fluorescence technique in breast cancer. Cancers 2010; 2: 713-720.

12. Liu J, Huang LP, Wang N, Chen P. Indocyanine green detects sentinel lymph nodes in earlybreast cancer. Journal of International Medical Research 2017; 45(2): 514-524.

13. Pitsinis V, Provenzano E, Kaklamanis L, Wishart GC, Benson JR. Indocyanine green fluorescence mapping for sentinel lymph node biopsy in early breast cancer. Surgical Oncology 2015; 24: 375379.

14. Grischke EM, Rohm C, Hahn M, Helms G, Bruker S, Wallwiener D. ICG Fluorescence Technique for the Detection of Sentinel Lymph Nodes in Breast Cancer: Results of a prospective open-label clinical trial. Geburtsh Frauenheilk 2015; 75: 935-940.

15. Samorani D, Fogacci T, Panzini I, Frisoni G, Accardi F, Ricci M, et al. The use of indocyanine green to detect sentinel nodes in breast cancer: a prospective study. EJSO 2015; 41: 64- 70.

16. Wishart GC, Loh SW, Jones L, Benson JR. A feasibility study (ICG-10) of indocyanine green (ICG) fluorescence mapping for sentinel lymph node detection in early breast cancer. EJSO 2012; 38: 651-656.

17. Alander JT, Kaartinen I, Laakso A, Patila T, Spillmann'T, Tuchin VV, et al. A review of indocyanine green fluorescent imaging in surgery. International Journal of Biomedical Imaging 2012; 26.
18. Veronesi U, Paganelli G, Viale G, Luini L, Zurrida S, Galimberti V, et al. Randomized comparison of sentinel-node biopsy with routine axillary dissection in breast cancer. N. Eng. J. Med. 2003; 349: 546-553.

19. Rubio IT, Klimberg VS. Techniques of sentinel lymph node biopsy. Semin. Surg. Oncol. 2001; 20: 214-223.

20. Giuliano AE. Sentinel lymphadenectomy in primary breast carcinoma: an alternative to routine axillary dissection. J. Surg. Oncol. 1996; 62: 75-77.

21. Linehan DC, Hill AD, Tran KN, Yeung H, Yeh SD, Borgen BI, et al. Sentinel lymph node biopsy in breast cancer: unfiltered radioisotope is superior to filtered. J. Am. Coll. Surg.1999; 188: 377-381.

22. Bass SS, Cox CE, Ku NN, Berman C, Reintgen DS. The role of sentinel lymph node biopsy in breast cancer. J. Am. Coll. Surg. 1999; 189: 183194.

23. Gipponi M, Bassetti C, Canavese G, Catturich A, Somma CD, Vecchio C, et al. Sentinel lymph node as a new marker for therapeutic planning in breast cancer patients. J. Surg. Oncol. 2004; 85: 102-111.

24. Chua B, Olivotto IA, Donald JC, Hayashi AH, Doris PJ, Turner LJ, et al. Outcomes of sentinel node biopsy for breast cancer in British Columbia 1996-2001. Am j Surg. 2003; 185:11826.

25. Cox CE, Bass SS, McCann CR, Ku NN, Berman C, Durand K, et al. Lymphatic mapping and sentinel lymph node biopsy in patients with breast cancer. Annu Rev Med. 2000; 51: 525-42.

26. Nano MT, Kollias J, Farshid G, Gill PG, Bochner M. Clinical impact of false-negative sentinel node biopsy in primary breast cancer. $\mathrm{Br}$ J Surg. 2002; 89:1430-4.

27. Chung A, Yu J, Stempel M, Patil S, Cody H, Montgomery L. Is the " $10 \%$ rule" equally valid for all subsets of sentinel-node-positive breast cancer patients. Ann. Surg. Oncol. 2008; 15: 2728 $-2733$.

28. Hojo 'T, Nagao T, Kikuyama M, Akashi S, Kinoshita T. Evaluation of sentinel node biopsy combined fluorescent and dye method and lymph flow for breast cancer. The Breast. 2010; 19(3): 210-213. 
29. Murawa D, Hirche C, Dresel S, Hunerbein M. Sentinel lymph node biopsy in breast cancer guided by indocyanine green fluorescence. Br. J. Surg. 2009; 96: 1289-1294. 